\title{
A few simple CFD models for flow simulation around groyne
}

\author{
Hriday Mani Kalita ${ }^{1, a}$, Arup Kumar Sarma ${ }^{2}$, Comingstarful Marthong ${ }^{1}$ and Deba Kumar Sarma ${ }^{3}$ \\ ${ }^{1}$ Civil Engineering Department, National Institute of Technology Meghalaya, Meghalaya, India, Pin: 793003 \\ ${ }^{2}$ Civil Engineering Department, Indian Institute of Technology Guwahati, Assam, India, Pin: 781039 \\ ${ }^{3}$ Mechanical Engineering Department, National Institute of Technology Meghalaya, Meghalaya, India, Pin: 793003
}

\begin{abstract}
The flow processes around a groyne structure is a complex and challenging topic in computational hydraulics and has brought attention of many researchers in the last few decades. In comparison to experimental studies, numerical modeling can provide more details about the flow characteristics at less cost. In the present work, performances of four simple finite difference schemes, i.e., Lax diffusive scheme, predictor corrector scheme, modified predictor corrector scheme and Beam and Warming scheme are compared in simulation of flow around groyne structure. The numerical results are validated with available experimental data and the best model is found out in terms of accuracy and efficiency.
\end{abstract}

\section{Introduction}

Any kind of structures those are constructed in a river for guiding/managing the river water for beneficial purpose of mankind are termed as river training structures. Groyne or spur dike is one of the most commonly used river training structures generally constructed from the river bank and extend towards the main channel flow. They are constructed for various purposes including, deflecting/repelling the flow away from bank for safety, increasing flow depth for smooth navigation, etc. Construction of these structures leads to blockage in the flow and always leads to changes in the local hydrodynamics as well as the morphology of the river. At the same time, the flowing water also imparts some effects on the groyne. These effects may be in the form of increment in the flow speed near the tip of the structure, development of local scouring around the structure due to pressure difference between the top and bottom water pressures near the structure. These local scouring many a time even may leads to failure of the structure itself. Moreover, the opposite river bank also faces high speed and shear stress upon construction of these structures. Therefore, before implementing a project deals with construction of groyne, it is always essential to conduct a model study to evaluate the probable effect of the groyne on the river as well as the reverse effect of the river on the structure itself. These model studies however, may be based on either experimental approaches or numerical approaches. In experimental studies, the whole groyne-river system is physically developed in some appropriate scales and experiments are conducted over this system. Ahmad [1] conducted one of the first experimental studies for simulating scouring process around groynes. In his study he observed that the water level remains constant upstream of the groyne, followed by a sudden drop near the tip and then gradually recovers along the downstream directions. However, he did not measure the flow velocity near the vicinity of the groyne. Rajaratnam and Nwachukwu [2] were the first researchers

\footnotetext{
${ }^{\mathrm{a}}$ Corresponding author : hridaymanikalita@gmail.com
} 
who tried to measure the flow velocity around a groyne. They conducted different set of experiments by changing the shape of groyne, flow depth, etc., and observed that groyne causes significant disturbances to the flow for a short distance upstream of it and for a longer distance downstream of it. From that time onward, several experimental studies were carried out by numerous researchers [3-4] for experimental simulation of groyne. However, these experimental models are subjected to some shortcomings like, high investment cost, scale issues, time consuming and limited measuring points. It has been observed that numerical models have the capacity to provide more details than physical models at low cost. Regarding the numerical simulation, the shallow water equations [5] can reasonably represent the flow characteristics around a groyne. In the recent decades, due to advancement in the computer power, researchers have also started using numerical models in this regard. Tingsanchali and Maheswaran [5] developed one of the first numerical models to simulate the flow of water around a groyne by solving the governing shallow water equations using a finite difference method. After that several two dimensional (2D) and three dimensional (3D) numerical models $[6,7]$ have been proposed by many investigators to replicate the complex flow processes around groyne. However, the flow processes around a groyne is very complex and therefore before using the numerical results for practical purposes a detail validation of these results with experimental data is still required. From the literature it appears that even though a large number of numerical models are available for flow simulation around groyne very less attention was given for comparing performances of different models. In this work four simple finite difference numerical models are presented for comparing and assessing the performances of the respective models on the basis of available flume data.

\section{Governing equations and numerical techniques}

\subsection{Governing equations}

The governing shallow water equations can be derived by vertically integrating the Navier Stokes equations over the flow depth under the assumptions of hydrostatic pressure distribution and small bottom slope. The governing equations are transformed from the physical coordinate system (x,y) to a boundary fitted coordinate system $(\xi, \eta)$ to make the numerical model compatible with complex boundaries and can be written as [8];

$$
P_{t}+Q_{\xi}+R_{\eta}+S=0
$$

where

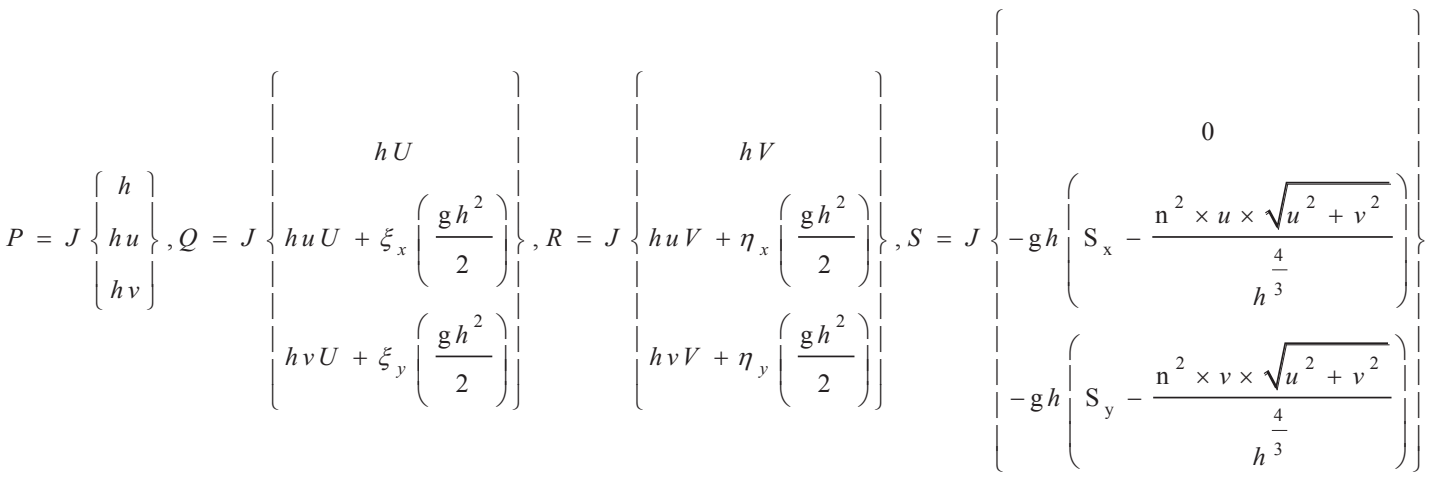


$h$ is depth of flow, $u, v, U, V$ are velocity components in $x, y, \xi$ and $\eta$ directions, respectively, $\mathrm{g}$ is the acceleration due to gravity, $\mathrm{S}_{\mathrm{x}}$ and $\mathrm{S}_{\mathrm{y}}$ are bed slopes is $\mathrm{x}$ and $y$ direction, respectively, $\mathrm{n}$ is Manning's roughness coefficient. The jacobian $J$ and the metrices $\left(\xi_{x}, \xi_{y}, \eta_{x}, \eta_{y}\right)$ are results of grid transformation and are calculated using central finite difference approximations [8].

\subsection{Numerical solution of the governing equations}

The governing equations presented by equation (1) constitute a system of mixed hyperbolic partial differential equations and are difficult to solve analytically without considering large number of approximations. Therefore, these equations are mostly solved using numerical techniques. In this work four different numerical techniques based on finite difference approximations are used in this regard and are presented in the subsequent sections.

\subsubsection{Lax diffusive scheme}

Lax diffusive scheme is a finite difference scheme based on explicit approach and is first order in time and second order accurate in space. The major advantages of this scheme are, single step in nature leading to less computational time requirement and no need of artificial damping/viscosity. Applying finite difference formulation of Lax diffusive scheme for the equation (1) yields [9];

$$
\begin{aligned}
& P_{i, j}^{k+1}=\frac{1}{4}\left(P_{i, j+1}^{k}+P_{i, j-1}^{k}+P_{i+1, j}^{k}+P_{i-1, j}^{k}\right)-\frac{\Delta t}{2 \Delta \xi}\left(Q_{i, j+1}^{k}-Q_{i, j-1}^{k}\right)-\frac{\Delta t}{2 \Delta \eta}\left(R_{i+1, j}^{k}-R_{i-1, j}^{k}\right) \\
& -\frac{\Delta t}{4}\left(S_{i, j+1}^{k}+S_{i, j-1}^{k}+S_{i+1, j}^{k}+S_{i-1, j}^{k}\right)
\end{aligned}
$$

where, $\Delta \xi$ is the grid spacing in flow direction designated by the subscript $j, \Delta \eta$ is the grid spacing in transverse direction designated by the subscript $i, \Delta t$ is the grid spacing in the time axis (time step) designated by the superscript $k$.

\subsubsection{Mac-Cormack predictor corrector scheme}

Mac-Cormack predictor corrector scheme is another explicit finite difference scheme which is second order accurate in both time and space. This technique is composed of two steps called predictor and corrector steps. Using backward difference in the predictor step an initial solution is obtained which is further corrected in the corrector step using forward difference. Final solution is obtained by taking average of these predicted and corrected solutions. Some of the advantages associated with this scheme are, capacity to handle mixed sub and super critical flow and efficient simulation of bore or shock waves. The governing equations can be discretized with this scheme as [9];

$$
\begin{aligned}
P_{i, j}^{p} & =P_{i, j}^{k}-\frac{\Delta t}{\Delta \xi}\left(Q_{i, j}^{k}-Q_{i, j-1}^{k}\right)-\frac{\Delta t}{\Delta \eta}\left(R_{i, j}^{k}-R_{i-1, j}^{k}\right)-\Delta t S_{i, j}^{k} \\
P_{i, j}^{c} & =P_{i, j}^{k}-\frac{\Delta t}{\Delta \xi}\left(Q_{i, j+1}^{k}-Q_{i, j}^{k}\right)-\frac{\Delta t}{\Delta \eta}\left(R_{i+1, j}^{k}-R_{i, j}^{k}\right)-\Delta t S_{i, j}^{p} \\
P_{i, j}^{k+1} & =\frac{1}{2}\left(P_{i, j}^{p}+P_{i, j}^{c}\right)
\end{aligned}
$$

\subsubsection{Modified predictor corrector scheme}

The governing equations presented above have an inherent property of signal propagation i.e., information comes only from the upstream direction in case of super critical flow and information 
comes from both upstream and downstream in case of subcritical flow. Therefore, in this modified version of the predictor corrector scheme backward finite difference is used in predictor step and forward finite difference is used in corrector step for subcritical region. However, for supercritical region, both steps employ backward difference to eliminate erroneous influence from the downstream flux on the computed values. This simple technique has made this scheme efficient in handing mixed flow regime. The governing equations can be discretized as follows;

$$
P_{i, j}^{p}=P_{i, j}^{k}-\frac{\Delta t}{\Delta \xi}\left(Q_{i, j}^{k}-Q_{i, j-1}^{k}\right)-\frac{\Delta t}{\Delta \eta}\left(R_{i, j}^{k}-R_{i-1, j}^{k}\right)-\Delta t S_{i, j}^{k}
$$

a) If $\sqrt{u^{2}+v^{2}}<\sqrt{g h}$, subcritical flow region,

$$
P_{i, j}^{c}=P_{i, j}^{k}-\frac{\Delta t}{\Delta \xi}\left(Q_{i, j+1}^{k}-Q_{i, j}^{k}\right)-\frac{\Delta t}{\Delta \eta}\left(R_{i+1, j}^{k}-R_{i, j}^{k}\right)-\Delta t S_{i, j}^{p}
$$

b) If $\sqrt{u^{2}+v^{2}}>\sqrt{g h}$, supercritical flow region,

$$
P_{i, j}^{c}=P_{i, j}^{p}
$$

\subsubsection{Beam and Warming scheme}

Beam and Warming scheme is an implicit finite difference scheme and second order accurate in both time and space. The major advantages of this scheme are its non-iterative nature and quick converging capacity. Here, the solution advances in a two-step sequence and each step requires solution of a block-tridiagonal system of equations. In the present work Thomas algorithm is used for efficient solution of the block-tridiagonal systems. The equations solved in $\xi$ and $\eta$ directions and the final values at the next time level can be computed as [9];

$$
\begin{gathered}
\left(\mathrm{I}+\frac{2 \Delta t}{3} \frac{\partial}{\partial \xi} A^{k}\right) P^{*}=-\Delta t \frac{3}{2}\left(\frac{\partial Q}{\partial \xi}+\frac{\partial R}{\partial \eta}+S\right)^{k}+\frac{1}{3}\left(P^{k}-P^{k-1}\right) \\
\left\{\mathrm{I}+\frac{2 \Delta t}{3}\left(\frac{\partial}{\partial \eta} B+C\right)\right\} P^{* *}=P^{*} \\
P_{i, j}^{k+1}=P_{i, j}^{k}+P_{i, j}^{* *}
\end{gathered}
$$

\subsubsection{Stability and Artificial viscosity}

The stability of the present models is represented using the Courant-Friedrichs-Lewy (CFL) criteria which restricts the time step value as;

$$
\Delta t=\frac{C r \times \min \times \min (\Delta x, \Delta y)}{\max [(u+\sqrt{g h}),(v+\sqrt{g h})]}
$$

where $\mathrm{Cr}$ is the Courant number and its value is limited to one for explicit schemes.

Another issue that arises while using second order accurate schemes is the dispersion errors and can be effectively removed by using artificial viscosity [9]. In the present work, the standard and the modified predictor corrector methods use a technique proposed by Jameson et al. [10] and the Beam and Warming methods uses a technique proposed by Klonidis and Soulis [11]. 


\section{Application of the models for flow simulation around groyne}

In order to appraise the performances of the present numerical schemes these are used to replicate the experimental study done by Rajaratnam and Nwachukwu for flow around groyne. In their study the flow channel was straight and rectangular in shape. The groyne considered was an aluminium plate of length $0.15 \mathrm{~m}$. The numerical models have been developed for an area extending from $1.8 \mathrm{~m}$ upstream to $3.6 \mathrm{~m}$ downstream of the groyne. A finite difference grid of size $97 \times 45$ is created for the flow domain. Manning's roughness value for the channel was 0.01 . An upstream flow of 0.045 cumec and a downstream depth of $0.189 \mathrm{~m}$ were used as boundary conditions. The channel bank and the groyne are simulated as reflective boundary technique. The $C r$ value used for the explicit schemes is 0.83 and the same for the implicit scheme is 2.2 . Figures $1 \mathrm{a}, 1 \mathrm{~b}, 2 \mathrm{a}$ and $2 \mathrm{~b}$ show the velocity vector plots for the Lax diffusive, predictor corrector, modified predictor corrector and Beam and Warming schemes, respectively. It appears from the results that the diffusive scheme produces a very small recirculation zone downstream of the groyne. It may be due to excessive diffusion associated with the scheme. However, a clear recirculation zone ending at $3.6 \mathrm{~m}$ is observed for the rest schemes exactly matching with the experiment.
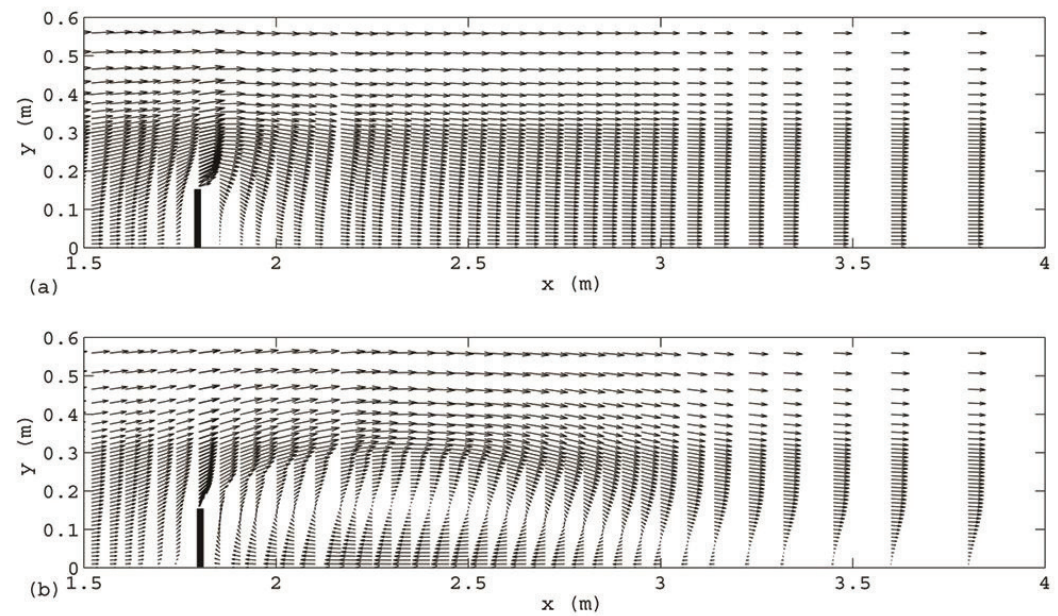

Figure 1 Velocity vector plot: a) Lax diffusive scheme, b) Predictor corrector scheme

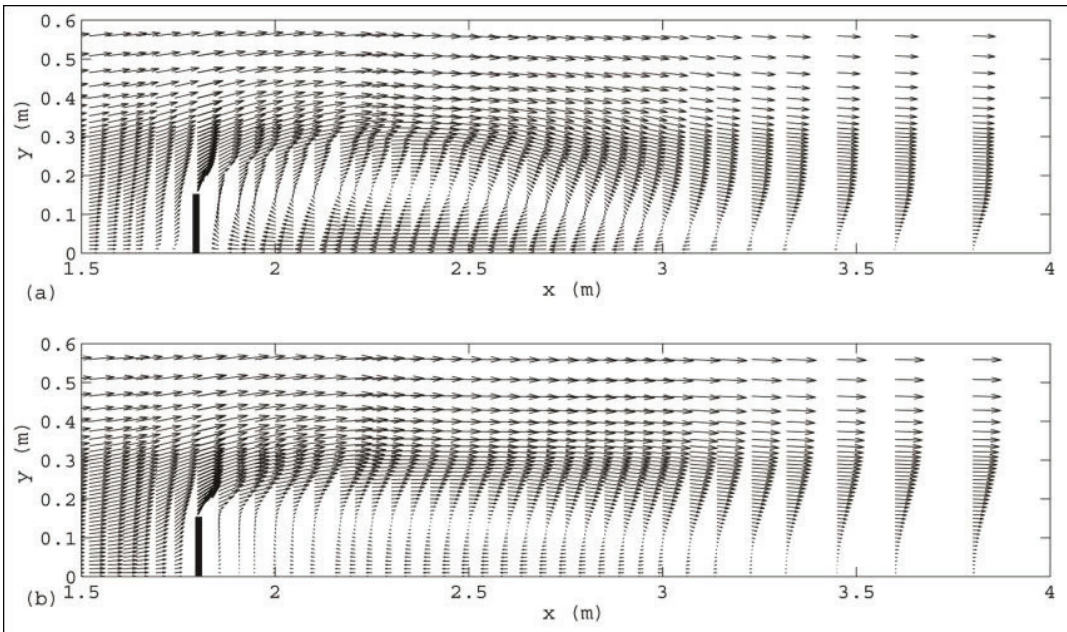

Figure 2 Velocity vector plot: a) Modified predictor corrector scheme, b) Beam and Warming scheme 
Apart from the recirculation zone comparison, the resultant velocity is also compared with the experimental data. For this purpose, the resultant velocity (W) is first non-dimensionalized with respect to the upstream velocity of $0.253 \mathrm{~m} / \mathrm{s}$. Figures $3 \mathrm{a}, 3 \mathrm{~b}, 3 \mathrm{c}$ and $3 \mathrm{~d}$ show the comparison of simulated flow speed and experimental flow speed at four horizontal lines at distances of $0.15,0.225$, 0.4 and $0.6 \mathrm{~m}$ from the southern bank, respectively. From these results it can be concluded here that the Beam and Warming scheme has the good potential for accurate simulation of flow characteristics around groyne.
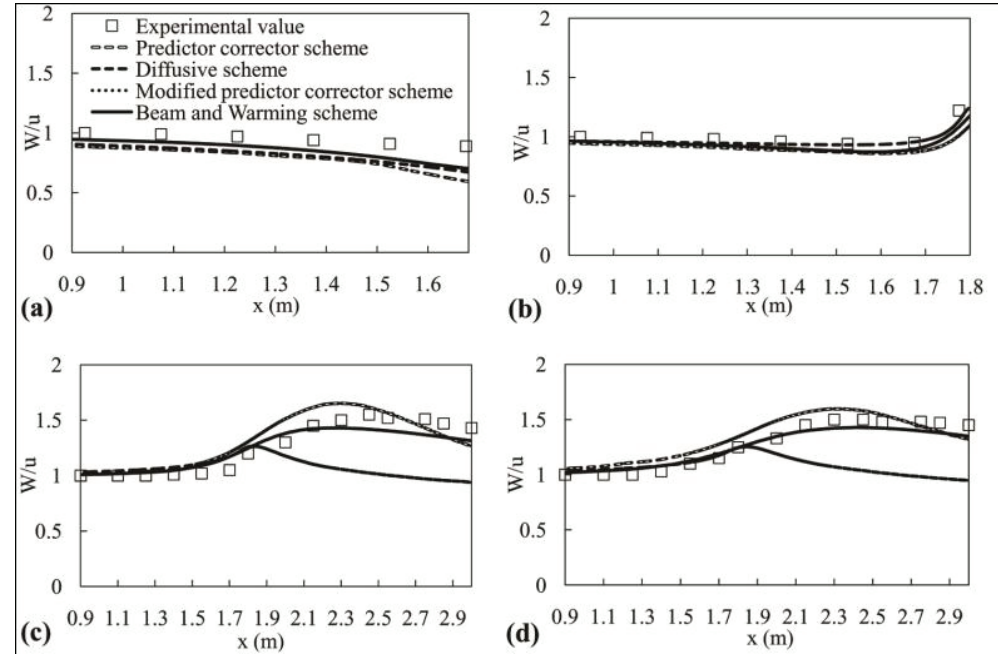

Figure 3 Comparison of flow speed: a) $y=0.15 \mathrm{~m}$, b) $0.225 \mathrm{~m}, \mathrm{c}) 0.4 \mathrm{~m}$, d) $0.6 \mathrm{~m}$

\section{Conclusions}

In this study, four simple finite difference based models, i.e., Lax diffusive, predictor corrector, modified predictor corrector and Beam and Warming, are developed to simulate the flow characteristics around groyne within flowing water. In order to check the capability of the developed models, the results are compared with an available experimental data. It appears from the results that the Lax scheme is not suitable for the affirmed task as it contains excessive diffusion. Out of the rest three schemes, Beam and Warming scheme is found to very much accurate in flow simulation around groyne. Moreover, this scheme also remains stable for higher values of temporal steps.

\section{References}

1. M. Ahmad, Minnesota Int. Hydraul. Convention (1953)

2. N. Rajaratnam, B. Nwachukwu, J. Hydraul. Eng. 109 (1983)

3. R.A. Kuhnle, C.V. Alonso, F.D. Shields, J. Hydraul. Eng. 128 (2002)

4. A. Safarzadeh, S.A.A. Salehi, A.R. Zarrati, J. Hydraul. Eng. 142 (2016)

5. T. Tingsanchali, S. Maheswaran, J. Hydraul. Eng. 116 (1990)

6. A. Molinas, Y.I. Hafez, J. fluids structures 14 (2000)

7. J. Yazdi, H. Sarkardeh, H.Md. Azamathulla, A.Ab. Ghani, Int. J. River Basin Manag. 8 (2010)

8. D.A. Anderson, J.D. Tannehill, R.H. Pletcher, Computational Fluid Mechanics and Heat Transfer (McGraw-Hill, New York, 1984)

9. M.H. Chaudhry, Open channel flow (Prentice-Hall Inc, Englewood Cliffs, 2008)

10. A. Jameson, W. Schmidt, E. Turkel, AIAA 14th Fluid And Plasma Dynamics Conference (1981)

11. A.J. Klonidis, J.V. Soulis, J. Hydraul Res. 39 (2001) 